\title{
Magnetic Analysis of the MQXF Quadrupole for the High Luminosity LHC
}

\author{
S. Izquierdo Bermudez, L. Fiscarelli, G. Ambrosio, H. Bajas, G. Chlachidze, P. Ferracin, J. Di Marco, S. Stoynev, \\ E. Todesco, G.L. Sabbi, G. Vallone.
}

\begin{abstract}
The high-luminosity upgrade of the large hadron collider (HL-LHC) requires new high-field and large-aperture quadrupole magnets for the low-beta inner triplets (MQXF). The US Hilumi-LHC Accelerator Upgrade (HL-LHC AUP) and CERN are jointly developing a $150 \mathrm{~mm}$ aperture $\mathrm{Nb}_{3} \mathrm{Sn}$ magnet. Due to the large beam size and orbit displacement in the final focusing triplet, MQXF has challenging field quality targets at collision energy. Magnetic measurements have been performed both at ambient and cryogenic temperatures in the four short models built and tested. This paper presents the magnetic analysis, comparing field measurements with the expectations and the field quality requirements. The analysis is focused on the geometrical harmonics and iron saturation effect, including three dimensional effects and transfer function repeatability. Persistent currents and dynamic effects are also discussed.
\end{abstract}

Index Terms - High Luminosity LHC, Field Quality, Magnetic Measurements, High Field $\mathrm{Nb}_{3} \mathrm{Sn}$ Magnet.

\section{INTRODUCTION}

$\mathbf{T}$ HE High Luminosity upgrade of the Large Hadron Collider (Hilumi-LHC) foresees the replacement of the low- $\beta$ inner triplet quadrupoles in the high luminosity interaction regions [1]. CERN and HL-LHC AUP (Hilumi-LHC Accelerator Upgrade Project) are developing together a $150 \mathrm{~mm}$ diameter aperture and $132.6 \mathrm{~T} / \mathrm{m}$ gradient $\mathrm{Nb}_{3} \mathrm{Sn}$ quadrupole, called MQXF [2]. The magnet will be produced in two lengths, MQXFA and MQXFB, with a magnetic length of $4.2 \mathrm{~m}$ and $7.15 \mathrm{~m}$ respectively. A series of short models (MQXFS), with a magnetic length of $1.2 \mathrm{~m}$, is being manufactured and tested. The first MQXF short model (MQXFS1) was assembled in LBNL [3] and tested at FNAL [4]-[5], using two coils produced by LARP (coils 3 and 5) and two coils pro-

Manuscript receipt and acceptance dates will be inserted here. This work was supported by the High Luminosity LHC Project at CERN and by the DOE through the U.S. LHC Accelerator Research Program. (Corresponding author: S. Izquierdo Bermudez)

S. Izquierdo Bermudez, is with CERN, 1211 Geneva, Switzerland, and also with the Escuela Tecnica Superior de Ingenieros Industriales (ETSII), Universidad Politecnica de Madrid (UPM), 28006 Madrid, Spain (e-mail: susana.izquierdo.bermudez@cern.ch)

P. Ferracin, H. Bajas, L. Fiscarelli, E. Todesco and G. Vallone are with CERN, 1211 Geneva, Switzerland.

G. Ambrosio, G. Chlachidze, S. Stoynev and J. Di Marco are with Fermi National Accelerator Laboratory (FNAL), Batavia, IL 60510 USA.

G.L. Sabbi and X. Wang are with Lawrence Berkeley National Laboratory (LBNL), Berkley, CA 94720 USA.

Color versions of one or more of the figures in this paper are available online at http://ieeexplore.ieee.org.

Digital Object Identifier will be inserted here upon acceptance.
TABLE I

COILS AND CONDUCTOR LAYOUT ASSEMBLED IN MQXFS

\begin{tabular}{ccccc}
\hline \hline & MQXFS1a/b/c & MQXFS3a/b & MQXFS5a & MQXFS4a \\
\hline & $\begin{array}{c}\text { First } \\
\text { Generation } \\
\text { Design }\end{array}$ & $\begin{array}{c}\text { Second } \\
\text { Generation } \\
\text { Design }\end{array}$ & $\begin{array}{c}\text { Second } \\
\text { Generation } \\
\text { Design }\end{array}$ & $\begin{array}{c}\text { Second } \\
\text { Generation } \\
\text { Design }\end{array}$ \\
\hline Q1 & $103^{1}$ & $106^{1}$ & $203^{3}$ & $108^{2}$ \\
Q2 & $3^{2}$ & $7^{2}$ & $205^{3}$ & $110^{2}$ \\
Q3 & $104^{1}$ & $105^{1}$ & $204^{3}$ & $109^{2}$ \\
Q4 & $5^{2}$ & $107^{1}$ & $206^{3}$ & $111^{2}$ \\
\hline \hline
\end{tabular}

${ }^{1}$ RRP $132 / 169 ;{ }^{2}$ RRP $108 / 127 ;{ }^{3}$ PIT 192

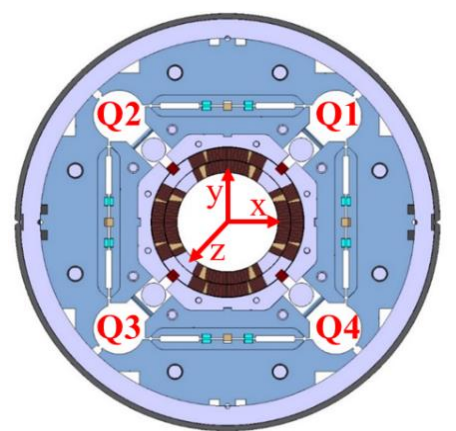

Fig. 1. MQXF magnet viewed from the lead end including coordinate axis for magnetic measurements and quadrant naming convention.

duced by CERN (coils 103 and 104). The rest of the short models (MQXFS3, MQXFS4 and MQXFS5) have been tested and assembled at CERN [6]-[7]. MQXFS3 had one coil produced by LARP (coil 7) and three coils produced by CERN (coils 105-107). MQXFS4 and MQXFS5 are assembled with coils produced in the same manufacturing line (CERN) and using a unique type of conductor. Table I summarizes the coils tested in each assembly and their relative position in the magnet (see Fig. 1). Magnetic measurements of MQXFS1, MQXFS3 and MQXFS5 were reported in the past [8]-[11]. This paper presents the analysis of MQXFS4 and compares the results to the previously tested magnets.

The field quality in the aperture is described in a standard form of harmonics coefficients defined in a series expansion,

$$
B_{y}+i B_{x}=B_{2} \cdot 10^{-4} \sum\left(b_{n}+i a_{n}\right)\left(\frac{x+i y}{R_{r e f}}\right)^{n-1}
$$

where $B_{x}$ and $B_{y}$ are the field components in Cartesian coordinates, $B_{2}$ is the reference field, and $b_{n}$ and $a_{n}$ are the normalized harmonics coefficients at the references radius $R_{r e f}=50 \mathrm{~mm}$. The right-handed measurement coordinate system is defined with the $z$-axis at the center of the magnet aperture and pointing from return to lead end. Measurements at room temperature combine \pm currents to remove magnetization effects. 
TABLE II

TRANSFer Function [T/M/KA] In The Magnet Straight SeCtion

\begin{tabular}{ccc}
\hline \hline & After loading & Nominal \\
\hline & $\mathrm{RT}$ & $1.9 \mathrm{~K}$ \\
\hline ROXIE & 8.840 & 8.051 \\
ROXIE + Deformation & 8.860 & 8.134 \\
MQXFS1a & 8.865 & 8.145 \\
MQXS3a & 8.839 & n. a. \\
MQXS4a & 8.820 & 8.130 \\
MQXS5a & 8.823 & 8.146 \\
\hline \hline
\end{tabular}

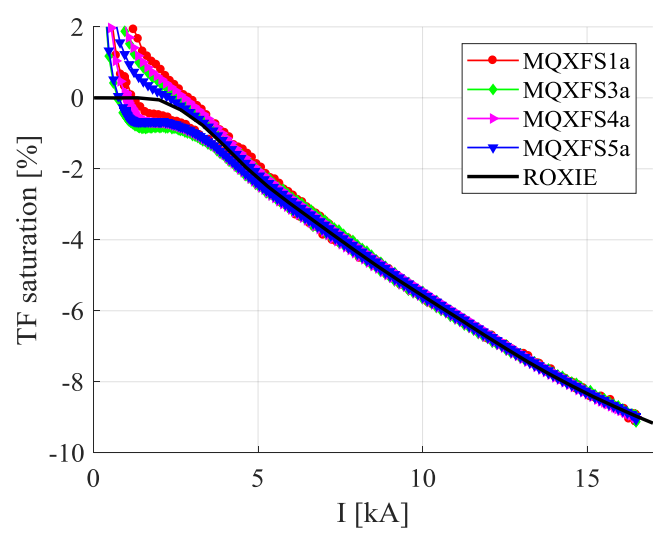

Fig. 2. Current dependence of the transfer function measured during a machine cycle to nominal current compared to ROXIE model.

\section{TRANSFER FUNCTION AND INTEGRAL FIELD}

The magnetic transfer function (TF) is defined as the ratio between the gradient and the magnet current. Table II summarizes the transfer function in the magnet straight section for all short models tested at room temperature after room temperature loading and at nominal operation conditions. The uncertainty of the measurements is on the order of $0.2 \%$. Accurate measurements on the absolute value of the transfer function at nominal current are not available for MQXFS3a. A specific cycle to precisely measure the main field was done in MQXFS3c but the magnet did not reach stable operation at nominal current. Nevertheless, the iron saturation effect in the transfer function can be evaluated from the standard machine cycle performed in MQXFS3a up to nominal current. Measurements are compared to computed values with ROXIE [14] using the nominal coil geometry at room temperature. The influence of coil deformation due to loading, cool down and powering is analyzed imposing in ROXIE the deformed geometry computed in ANSYS. At nominal operation conditions, the transfer function is within 15 units the expected values for all the magnets when including the coil deformation effect. The longitudinal variation of the transfer function along the magnet straight section after magnet loading at room temperature is \pm 2 units in MQXFS3 and MQXFS5, \pm 5 units in MQXFS1 and MQXFS4.

Figure 2 shows the relative change of the measured transfer function as a function of the magnet current in a central segment of the magnet, and compare it with the expected iron saturation effect from 2D magnetic computations. Measured
TABLE III

Magnetic Length [Mm] At Nominal CuRRent. Computed Values AsSUME 3 MM/M OF LONGITUDINAL THERMAL CONTRACTION

\begin{tabular}{ccc}
\hline \hline & Measured & Computed \\
\hline MQXFS1 & 1198 & 1192 \\
MQXS3 & 1204 & 1198 \\
MQXS4 & 1203 & 1198 \\
MQXS5 & 1203 & 1198 \\
\hline \hline
\end{tabular}

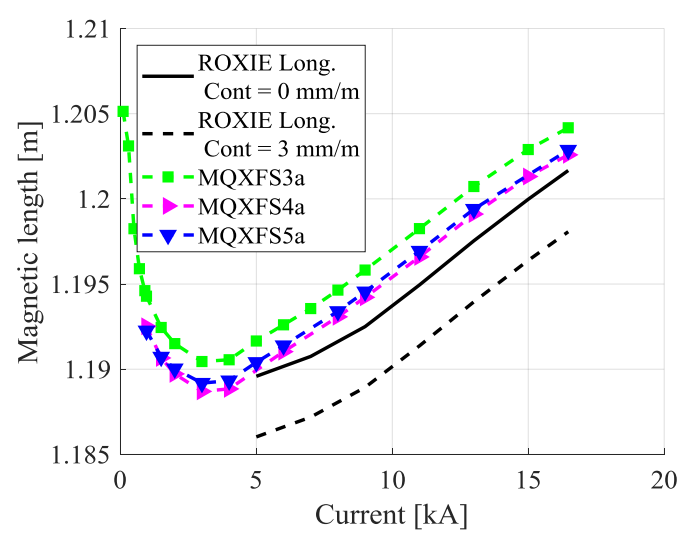

Fig. 3. Current dependence of the transfer function measured during a stair step cycle to nominal current compared to ROXIE model.

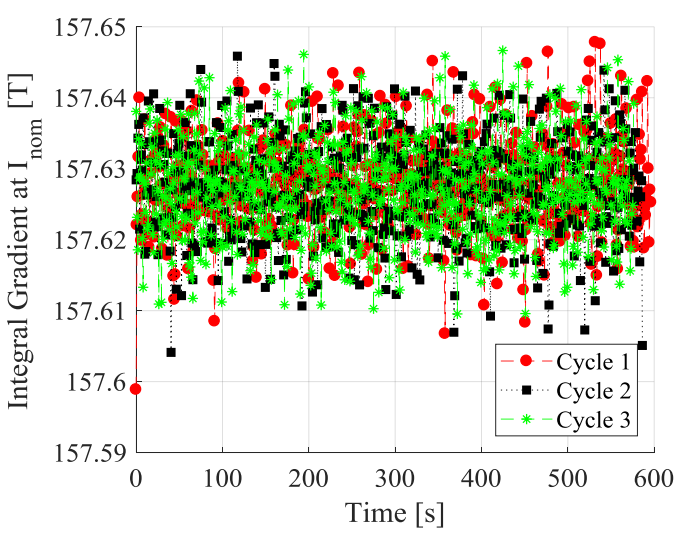

Fig. 4. Cycle to cycle reproducibility on the integral field for MQXFS4a during three consecutive machine cycles to nominal current.

and computed values are within 5 units at all current levels, with a decrease on the transfer function by $\sim 9 \%$ form injection to nominal current. Iron saturation effect is also visible in the magnetic length, defined as the ratio between the integral field and the main field in the straight section. Figure 3 compares the magnetic length as a function of the current for the magnets measured at CERN with the model predictions. Measurements are done following a stair-step cycle. A precycle with a flattop current of $16.47 \mathrm{kA}$ and a reset current of 100 A preceded the measurements. The model correctly predicts the iron saturation effect on the magnetic length but under-estimates the absolute value by $5 \mathrm{~mm}$ when assuming a longitudinal thermal contraction of $3 \mathrm{~mm} / \mathrm{m}$. Table III summarizes the magnetic length at nominal operation conditions, showing a magnet to magnet reproducibility of $1 \mathrm{~mm}$. The difference between MQXFS1 and the rest of the magnets is expected due to the differences in coil geometry between the first and second generation design.

A repeatable and stable behavior at collision energy is critical, with a tight beam dynamics requirement of \pm 0.5 units. Figure 4 shows the integral gradient at collision in three 
TABLE IV

AVERAge Harmonics [Units] IN THE StRAIGHT SECTION IN MQXFS4

\begin{tabular}{c|cc|cc|cc}
\hline \hline & \multicolumn{2}{|c|}{ Before } & \multicolumn{2}{c|}{ After } & \multicolumn{2}{c}{$\mathrm{I}=16.47 \mathrm{kA}$} \\
$\mathrm{n}$ & $b_{n}$ & $a_{n}$ & $b_{n}$ & $a_{n}$ & $b_{n}$ & $a_{n}$ \\
\hline 3 & -3.44 & 0.05 & -4.80 & 0.71 & -2.61 & 1.18 \\
4 & -0.78 & 2.49 & 0.53 & 4.09 & 0.55 & 4.75 \\
5 & 2.35 & 0.82 & 2.37 & 0.70 & 3.16 & 0.21 \\
6 & -4.82 & 0.37 & -3.47 & 0.38 & -3.78 & 0.37 \\
7 & -0.26 & -0.15 & -0.34 & -0.13 & -0.32 & 0.18 \\
8 & -0.02 & 0.55 & 0.02 & 0.59 & 0.01 & 0.78 \\
9 & 0.17 & 0.08 & 0.20 & 0.12 & 0.27 & 0.12 \\
10 & 0.38 & 0.03 & 0.48 & -0.01 & 0.09 & -0.01 \\
\hline \hline
\end{tabular}

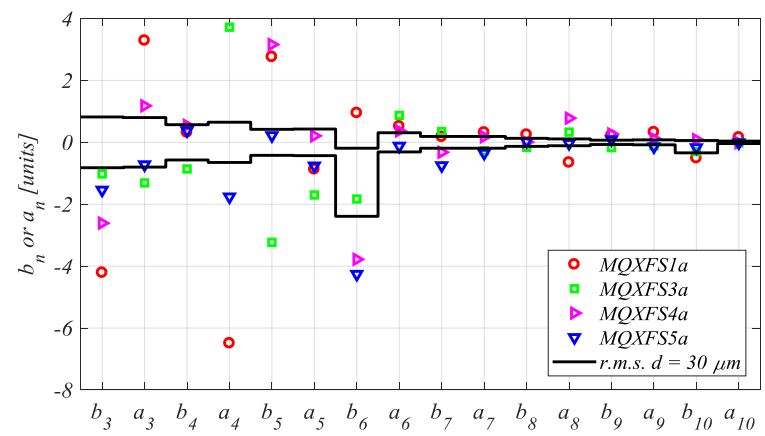

Fig. 5. Average measured harmonics in the magnet straight section at nominal field compared with target field quality (target field quality computed assuming a random displacement of the coil block of $30 \mu \mathrm{m}$ ).

consecutive machine cycles. A pre-cycle with a flattop current of $16.47 \mathrm{kA}$ and a reset current of 100 A preceded the measurements. The reproducibility and stability of the integral field is within the noise level of the measurements ( \pm 1 units). On average, the cycle to cycle reproducibility is better than 0.1 units.

\section{GEOMETRIC FIELD ERRORS}

Due to the large beam size and orbit displacement in the final focusing triplet, field errors at high energy are of primary importance. Geometric field errors of MQXFS1, MQXFS3 and MQXFS5 were extensively studied in [11]. The measured geometric field errors, averaged in the magnet straight section for MQXFS4 are summarized in Table IV. As observed in previous magnets, there is good correlation between room temperature and $1.9 \mathrm{~K}$ measurements and the overall strength and longitudinal structure of the harmonics is not fundamentally altered from the initial assembly before loading to powering [9]. Figure 5 shows the harmonics at nominal field averaged over the axial straight section and compares them to the target field quality based on a random error conductor position of $30 \mu \mathrm{m}$.

1) Systematic allowed field errors

A summary of the allowed multipoles at nominal field is provided in Table V. Measurements are compared to ROXIE computed values for the case the conductors are aligned to the inner diameter (ID) and to the outer diameter (OD) of the coil. The last two columns show the expected impact of the actual coil geometry (including coil shimming layout) and the coil deformation as computed in ANSYS. ID or OD alignment has a great impact on $b_{6}$. The impact of coil shimming and deformation is small compared to OD/ID alignment. MQXF design
TABLE V. Measured and Predicted Allowed Multipoles At Nominal CURRENT (INCLUDING IRON EFFECT) [UNITS].

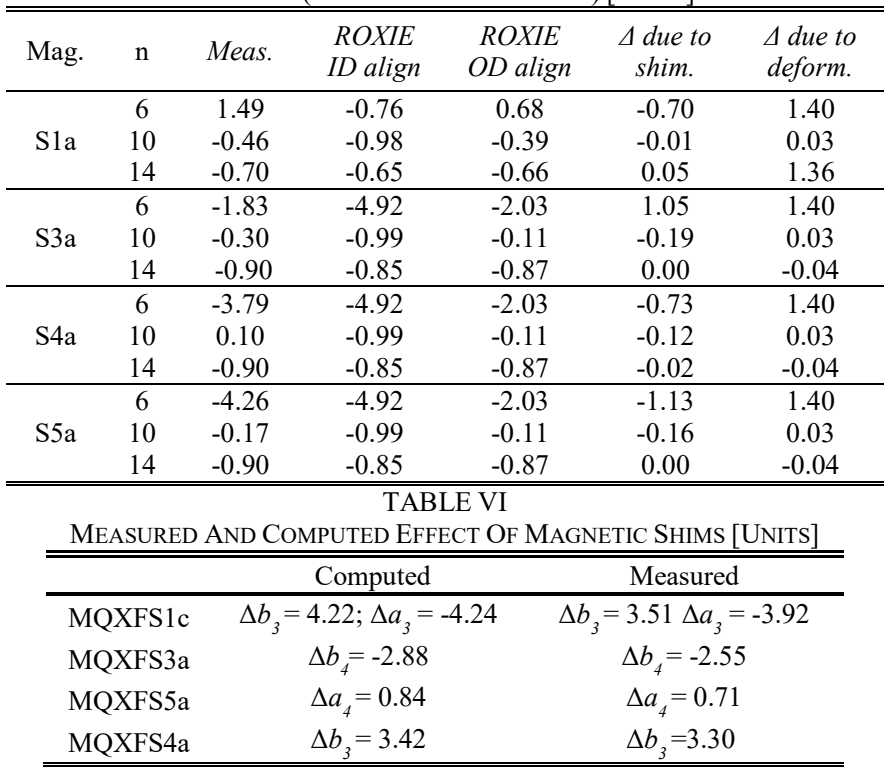

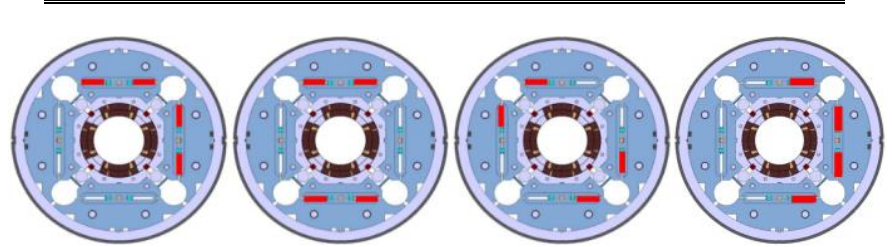

Fig. 6. Magnetic shim configuration from left to right in MQXFS1c, MQXF3a, MQXFS5a and MQXFS4a.

was optimized assuming the coil turns are aligned to the OD. Measured $b_{6}$ is in general lower than expected when aligning the coil turns to the OD, which could indicate that the conductors are partially aligned to the ID, consistent with the design assumptions for the LHC Main Quadrupoles [12]-[13]. The relative difference on $b_{6}$ between MQXFS3 and MQXFS4/5 can be explained by the differences in coil shimming. Measured $b_{14}$ is close to expected values.

In order to reach the target $b_{6}$ of $\sim 0$ units at collision energy, the plan is to fine tune the allowed harmonics increasing the pole insulation thickness before the beginning of the winding by $0.125 \mathrm{~mm}$ and reducing the mid-plane shim thickness by $0.125 \mathrm{~mm}$ before coil impregnation. A $\Delta b_{6}=+5.3$ units and $\Delta b_{10}=+0.3$ is expected. These actions take place during coil fabrication, and they allow to keep the same quantity of material in the same impregnation cavity.

\section{2) Systematic non-allowed field errors}

The azimuthal size for coils assembled in the short models is not uniform [15], requiring asymmetric coil shimming to compensate for dimensional errors and assure uniform preload. In the case of MQXFS4a, the expected harmonics due to asymmetric shimming are -0.3 units of $b_{3}, 0.3$ units of $a_{4}, 0.6$ units of $b_{5}$ and 0.2 units of $a_{5}$. Whereas in previous magnets asymmetric shimming could partially explain the measured field errors [11], non-allowed harmonics in MQXFS4a are considerably larger than expected shimming effect. At nominal field, $b_{4}$ is 4.7 units and $b_{5}$ is 3.2 units. Similar amplitude for these harmonics was measured in all previous magnets except MQXFS5. It is important to point out that in order to cope 
with the bubble formation in the inner layer quench heaters [16], each coil in MQXFS4a had a different inner layer quench heater configuration. Since the good alignment of the inner edge of the conductors is critical for field quality, different insulation lay-outs in the inner diameter of the coils is an additional source of errors not representative for series magnets.

Thanks to the good correlation between room temperature and $1.9 \mathrm{~K}$ measurements, non-allowed field errors can be partially compensated through ferromagnetic shims located in the yoke [14], [17]. The maximum correction capability is \pm 4.5 units of $b_{3}$ and $a_{3}, \pm 3$ units of $b_{4}$ and \pm 1 units of $a_{4}$. This technique has been used in all MQXF short models. Table VI compares the measured and expected variation of the multipoles and Fig. 6 shows the shimming configuration for the different assemblies. Measurements are within $10 \%$ the expected value.

\section{STRAND MAGNETIZATION EFFECT}

Figure 8 shows the measured $b_{6}$ as a function of the current for all measured magnets during a machine cycle. The cycle to cycle repeatability is better than 0.1 units. A geometric offset is applied to the measurements in order to have $b_{6}=0$ at collision energy. Measurements are compared to the model, showing good agreement. The change on $b_{6}$ from injection to nominal is 20 units for the MQXFS5 and 25-30 units for the rest of the magnets. The smaller magnetization in MQXFS5 is expected since the sub-element diameter of the superconducting filaments is $41 \mu \mathrm{m}$ (PIT 192 conductor), to be compared to $55 \mu \mathrm{m}$ for RRP $108 / 127$ and $50 \mu \mathrm{m}$ for RRP 132/169.

\section{Multipole DECAY}

The present understanding of the decay is that the local field changes due to current redistribution during a constant current plateau cause a decrease of the average filament magnetization [18]. Fig. 9 shows that this is not the case for the measurements on MQXFS3 and MQXFS4, i.e., the magnets built with RRP conductor. A net increase of the strand magnetization is observed. As soon as the field is ramped up again after the end of injection, the magnetization rapidly recovers the original hysteresis curve. The operation temperature has a very large

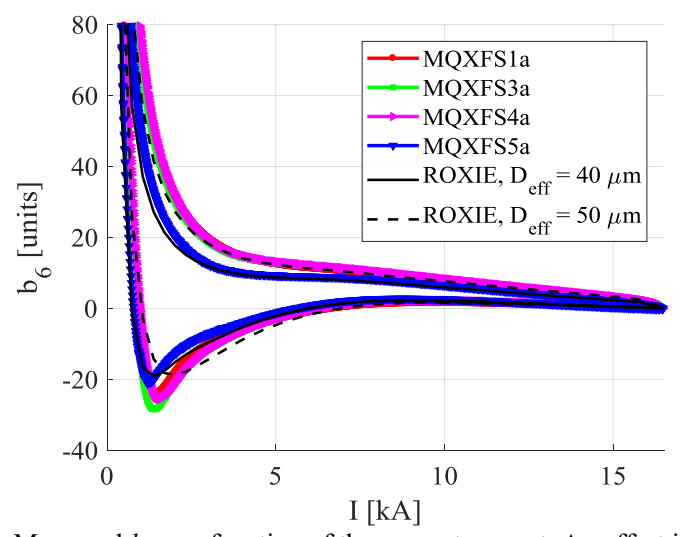

Fig. 8. Measured $b_{6}$ as a function of the magnet current. An offset is applied to all the measurements to have $b_{6}=0$ at collision energy.
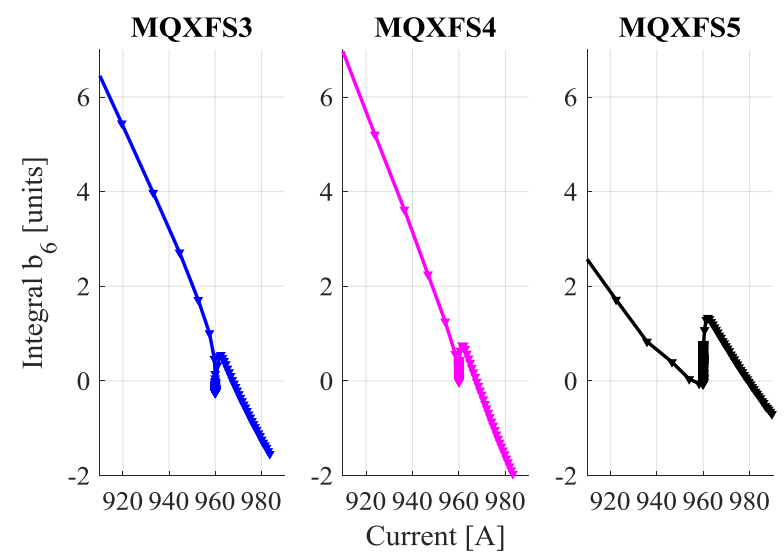

Fig. 9. Decay and snapback measured in the MQXFS magnets tested at CERN. The curve is vertically shifted so that $b_{6}=0$ at injection $(960 \mathrm{~A})$.

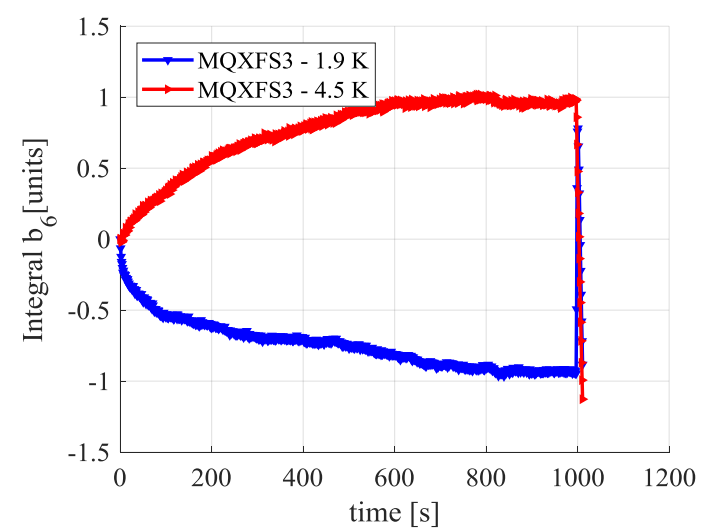

Fig. 10. Impact of the operation temperature on the integral decay of $b_{6}$.

impact in the decay, with an inversion on the sign of the decay at $4.3 \mathrm{~K}$ (see Fig. 10). This behavior is consistent with previous observations on the $11 \mathrm{~T}$ dipole [19], pointing to the idea that the state of magnetization of the filaments (including the effects of flux jumps) plays a major role. This effect is not observed in magnets tested in FNAL [20]. MQXFS5, the magnet built with PIT conductor, shows a very different behavior: during the injection plateau, a net decrease of the average filament magnetization is measured, followed by a very slow recovery of the initial magnetization curve.

\section{CONCLUSION}

Four MQXF short models have been built and tested. Magnetic measurements give an indication of a systematic $b_{6}$ far from the target of 0 units at collision energy. In order to reach the target, the plan is to increase the pole insulation thickness and reduce the mid-plane shim thickness by $0.125 \mathrm{~mm}$. Correction capabilities through magnetic shims have been extensively demonstrated, but they are not enough to correct the 2-6 units of $a_{4}$ and 1-3 units of $b_{5}$ observed in the short models. In order to cope with this larger non-allowed harmonics, the integral strength of the high order corrector magnets has been increased. MQXFS5, the only magnet assembled with coils following a uniform manufacturing process has a remarkable better field quality. Transfer function is in agreement with expectations and cycle to cycle repeatability is suitable for operation. 


\section{REFERENCES}

[1] G. Apollinari, I. Bejar Alonso, O. Brüning, M. Lamont, and L. Rossi, "High-Luminosity Large Hadron Collider (HL-LHC): Preliminary Design Report", CERN Yellow Reports Monographs, CERN, Geneva, 2015.

[2] P. Ferracin, et al., "Magnet design of the $150 \mathrm{~mm}$ aperture low- $\beta$ quadrupoles for the high luminosity LHC," IEEE Trans. Appl. Supercond., vol. 24, no. 3, Art. ID 4002306, 2004.

[3] H. Pan, et al., "Assembly Tests of the First Nb3Sn Low-beta Quadrupole Short Model for the Hi-Lumi LHC," IEEE Trans. Appl. Supercond., vol. 26, no. 4, pp. 4001705, 2016.

[4] G. Chlachidze, et al., "Performance of the first short model $150 \mathrm{~mm}$ aperture Nb3Sn quadrupole MQXFS for the High-Luminosity LHC upgrade," IEEE Trans. Appl. Supercond., vol. 27, no. 4, pp. 1-5, 2017.

[5] G. Chlachidze et al., "Summary of test results of MQXFS1 - the first short model $150 \mathrm{~mm}$ aperture $\mathrm{Nb}_{3} \mathrm{Sn}$ quadrupole for the high-luminosity LHC upgrade," IEEE Trans. Appl. Supercond., 2018.

[6] H. Bajas et al., "Test results of the short models MQXFS3 and MQXFS5 for the HL-LHC upgrade," IEEE Trans. Appl. Supercond., 2018

[7] H. Bajas et al., "Test results of the short models MQXFS4 for the HLLHC upgrade," IEEE Trans. Appl. Supercond., Submitted for publication, 2019

[8] J. DiMarco, et al., "Magnetic Measurements of the Nb3SN Model Quad (MQXFS) for the High-Luminosity LHC upgrade," IEEE Trans. Appl. Supercond., Vol. 27, no. 4, pp 90000105, 2017.

[9] S. Izquierdo Bermudez, et al., "Magnetic analysis of the $\mathrm{Nb}_{3} \mathrm{Sn}$ low- $\beta$ for the High Luminosity LHC," IEEE Trans. Appl. Supercond., vol. 27, no. 4, pp. 4001905, 2017.

[10] L. Fiscarelli, et al., "Magnetic Measurements on the First CERN-built Models of the Insertion Quadrupole MQXF for HL-LHC", IEEE Trans. Appl. Supercond., vol. 28, no. 3, pp. 4002605, 2018.

[11] S. Izquierdo Bermudez, et al., "Geometric Field Errors of Short Models for MQXF, the Nb3Sn low- $\beta$ Quadrupole for the High Luminosity LHC," IEEE Trans. Appl. Supercond., vol. 28, no. 3, pp. 4006306, 2018.

[12] W. Scandale, et al., "Field Quality in the LHC Main Quadrupole Prototype before the Cold Test", LHC Project Note 209, Dec. 1999.

[13] E. Todesco, et al., Analysis of the magnetic measurements at room temperature of the LHC main quadrupole prototypes", LHC Project Note 243, Nov. 2000.

[14] S. Russenschuck, "ROXIE: A computer code for the integrated design of accelerator magnets," in Proc. 6th Eur. Particle Accelerator Conf., 1999, pp. 2017-2019.

[15] J. Ferradas Troitino, et al., "Applied Metrology in the Production of Superconducting Model Magnets for Particle Accelerators," IEEE Trans. Appl. Supercond., vol. 28, no. 3, pp. 4002106, 2018.

[16] S. Izquierdo Bermudez, et al., "Overview of the Quench Heater Performance for MQXF, the $\mathrm{Nb}_{3}$ Sn Low- $\beta$ Quadrupole for the High Luminosity LHC," IEEE Trans. Appl. Supercond., vol. 28, no. 4, pp. 4008406, 2018.

[17] S. Izquierdo Bermudez, et al., "Second Generation Coil Design of the $\mathrm{Nb}_{3} \mathrm{Sn}$ low- $\beta$ Quadrupole for the High Luminosity LHC," IEEE Trans. Appl. Supercond., vol. 26, no. 4, pp. 4001105, 2016.

[18] M. Haverkamp, Ph.D. dissertation, Twente University, Enchede, 2003.

[19] S. Izquierdo Bermudez, et al., "Decay and Snapback in $\mathrm{Nb}_{3} \mathrm{Sn}$ Dipole Magnets", IEEE Trans. Appl. Supercond., vol. 27, no. 4, pp. 4002306, 2017.

[20] G. V. Velev, et al., "Summary of the Persistent Current Effect Measurements in $\mathrm{Nb}_{3} \mathrm{Sn}$ and $\mathrm{NbTi}$ Accelerator Magnets at Fermilab" IEEE Trans. Appl. Supercond., vol. 26, no. 4, pp. 4000605, 2016. 